\title{
An Immunoelectron Microscopic Comparison of Desmosomal Constituents and Hemidesmosomal Ones Originating from the Same Tissue of the Same Animal
}

\author{
Hisato Shida and Malcolm S. Steinberg* \\ Department of Biology, University of Yamanashi Medical School, Tamaho, Yamanashi 409-38, Japan and \\ *Department of Biology, Princeton University, Princeton, NJ 08544, USA
}

Key words: desmosome/hemidesmosome/immunoelectron microscopy/protein A-gold/epithelial cells

\begin{abstract}
The molecular constituents of desmosomes and hemidesmosomes were compared by examining bovine muzzle epidermis under immunoelectron microscopy using a postembedding method, first with antibodies prepared to four desmosomal antigens (DP1/2, DP3, DG1, DG2/3), followed by protein A-gold (PAG) complexes. The four antibodies showed almost negative labeling at hemidesmosomes as compared with the labeling observed at the desmosomes in the same tissue. By counting the number of PAG particles $/ 200 \mathrm{~m} \mu$ at hemidesmosomes and desmosomes, the above qualitative observation was confirmed quantitatively. These results support a new concept which has recently been proposed by several researchers that hemidesmosomes and desmosomes are immunochemically distinct.
\end{abstract}

The hemidesmosome is a kind of adhering junction which is ultrastructurally specialized at the region of the joint area between the epithelial cells and the thin layers of the underlying extracellular matrix called basal laminae. Although some researchers have pointed out an ultrastructural resemblance between hemidesmosomes and desmosomes (8), no conclusive evidence has yet been given as to whether or not their molecular constituents are the same.

In the early studies, desmosomal plaque proteins were pointed out as a common constituent of hemidesmosomes $(2,11)$. One of these papers showed clearly that hemidesmosomal plaques of bovine tongue mucosa were positively stained according to the immunoperoxidase technique with a guinea-pig antibody to desmoplakin I and II (synonyms of desmosomal proteins 1/2; DP1/2). After a couple of years, conflicting data were reported by several investigators $(6,19,24)$. These results included immunofluorescence analysis with antiDP antibodies and with bullous pemphigoid (BP) autoantibodies. The basement membrane zone (BMZ) which should include hemidesmosomes was not stained by the anti-desmoplakin antiserum, while BP sera stained only BMZ $(6,24)$. These findings were also confirmed on an immunoelectron microscopic level with the same antibody and a monoclonal antibody to desmosomal glycoprotein (DG) 1 (6). At nearly the same time, we reported experimental results on a systematic analysis by immunoelectron microscopy using four major desmosomal antigens, including antiDP $1 / 2$, in an abstract (19). In our research, we did not detect any common antigens between desmosomes and hemidesmosomes, even in the same tissue, by immunogold labeling using the postembedding procedure. Although our results with a polyclonal antibody to DP $1 / 2$ agree with the results achieved by Jones et al. (6), other investigators have referred to a weak positive reaction on DP1/2 at hemidesmosomes of the bovine nasal epithelium (10).

In an attempt to resolve the above problem, we carefully considered the following points in our experiments. 1) Near the basal region of epidermal cells, desmosomes which are close to hemidesmosomes can be observed. For the purpose of avoiding confusion regarding identification of hemidesmosomes, immunoelectron microscopy ought to be used rather than immunofluorescence microscopy. 2) In changing tissues, the crossreactivity of desmosomes in these tissues should first be checked using an antibody which has been prepared for the desmosomal antigen from bovine snout epidermal tissue. In our quantitative analysis by immunoelectron microscopy, the labeling intensity of desmosomes with the same antibody changed from tissue to tissue (16). For this reason, hemidesmosomes sould be checked in the same tissue as desmosomes. 3) Some of the most important proteins which can be linked with the adhesion of desmosomes will likely be the major desmosomal glycoproteins DG1, DG2/3, and DG4. To clarify the distinct property of hemidesmosomes, the presence of both DP and DG families should be investigated with immunoelectron microscopy. 4) Finally, for the purpose of immunocytochemical comparison between the hemidesmosomal and desmosomal constituents, polyclonal antibodies are preferable to monoclonal anti- 
bodies since a monoclonal antibody recognizes only one epitope in the large-size molecule.

In the present report, we have clarified the above concept that the hemidesmosome is immunochemically distinct and not simply half a desmosome by applying immunoelectron microscopy with four antibodies to desmosomal antigens, i.e., DP1/2, DP3, DG1, and DG2/3 of the bovine muzzle epidermis, using a postembedding staining procedure.

\section{MATERIALS AND METHODS}

Antibodies. To avoid confusion about the terminology of desmosomal components, the systematic naming system of Miller et al. (10) was employed in the present paper. Four monospecific polyclonal antibodies directed against $\mathrm{DP} 1 / 2$, DP3, DG1, and DG2/3 were used for immunoelectron microscopy. The method of production of antibodies has been described elsewhere (4). Specificity of the antibodies was demonstrated by immunoblot staining of the desmosomes isolated from bovine snout. All the antibodies showed basically monospecific patterns corresponding to the specific antigens (Fig. 1).

Immunoelectron microscopy. We have used JB-4 resin for immunoelectron microscopy $(20,21)$. Fixation and embedding in JB-4 resin were carried out as previously described. Briefly, a thin slice of fresh bovine muzzle was fixed with a mixture of $4 \%$ paraformaldehyde and $0.1 \mathrm{M}$ phosphate

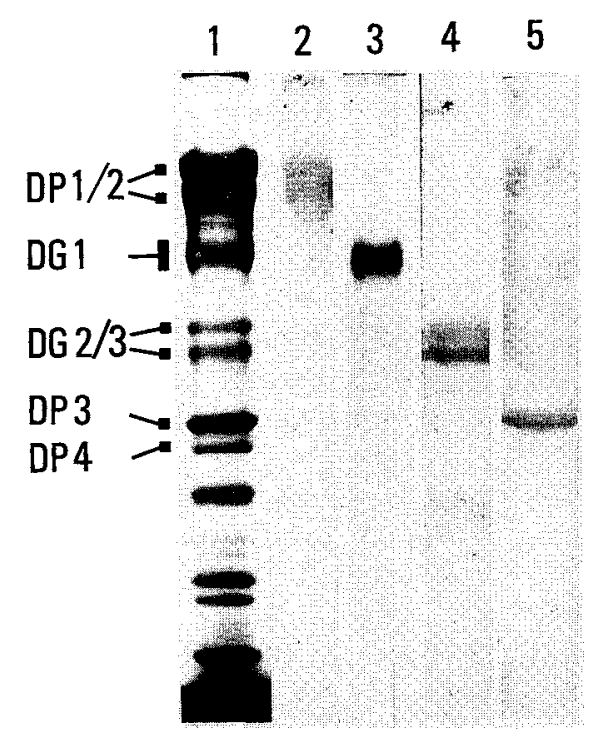

Fig. 1. Immunoblotting with four antibodies against desmosomal proteins. Isolated bovine muzzle desmosomes were subjected to sodium dodecyl sulfate-polyacrylamide gel electrophoresis and were transferred to Zeta-Probe (Bio-Rad Lab., CA 94804) for immunoblotting. Lane 1: Coomassie blue-stained strip of the gel used to generate the blots. Lane 2-5: Zeta-Probe blots labeled with rabbit antibodies directed against (lane 2) DP1/2; (lane 3) DG1; (lane 4) DG2/3; (lane 5) DP3. buffer (pH 7.3) for one $h$ at $4^{\circ} \mathrm{C}$. After dehydration with an ethanol series, the fixed tissue was infiltrated with JB-4 solution A (Polysciences Inc., PA) containing $0.9 \%$ of catalyst at $4^{\circ} \mathrm{C}$ overnight. The infiltrated sample was then transferred to a mixture of twenty parts freshly prepared JB-4A, one part methyl methacrylate, one part divinylbenzene, and one part JB-4B, which was placed into BEEM capsules and sealed with Parafilm. The resin was polymerized at $8^{\circ} \mathrm{C}$ for at least $6 \mathrm{~h}$ followed by UV irradiation in a curing chamber (Ladd Industries, Burlington, VT) at room temperature overnight.

In the immunocytochemical labeling, all procedures were conducted at room temperature. Ultrathin sections of tissue on grids were first placed in Dulbecco's phosphate-buffered saline prepared without calcium or magnesium salt (PBS-), and were then incubated in $0.5 \%$ BSA (fraction V, Sigma Chem. Co., St. Louis, MO) in PBS- for $30 \mathrm{~min}$ to block the nonspecific binding of antibodies. The sections were then incubated in a primary antibody or preimmune serum diluted in PBS-containing $0.5 \% \mathrm{BSA}$ for $6 \mathrm{~h}$. After being twice washed for 15 min each time in PBS-, the grids were incubated in $0.5 \%$ BSA in PBS- and then labeled with a suspension of PAG conjugates, whose average diameter was $12 \mathrm{~nm}$, for $1 \mathrm{~h}$. The labeled grids were next washed two more times with PBSfor 10 min each time, and were then washed once more with distilled water. Finally, the labeled sections were stained with a $1 \%$ aqueous solution of uranyl acetate for between 5 to 15 min. Thin sections were examined with a Hitachi H-600 or a JEOL 100CX transmission electron microscope at an acceleration voltage of $100 \mathrm{KV}$.

Quantification of the labeling intensity. Desmosomes and hemidesmosomes sectioned at right angles to the cell membrane were selected for quantitative analysis. Using photography set at $\times 330,000$ magnification, PAG particles on desmosomes or hemidesmosomes per $200 \mathrm{~m} \mu$ were counted up to 10 data points.

\section{RESULTS}

Immunoelectron microscopy obtained with $D P 1 / 2$. At the spinosum region of the bovine snout epidermis, the specific immunocytochemical labeling obtained with anti-DP1/2 antibody was restricted to the desmosomes. As shown in Fig. 2-b, this specific positive reaction was mainly observed around and on desmosomal plaques.

Using the same electron micrographs, we were able to examine the basal layer, which included both hemidesmosomes and desmosomes. While the labeling of desmosomes was positive, hemidesmosomes showed a negative reaction in the same field with anti-DP $1 / 2$ (Fig. 2-a).

Immunoelectron microscopy obtained with DP3, $D G 1$, and $D G 2 / 3$. The same tendency was observed with anti-DP3 antibody (Figs. 3-a, -b). This antibody showed a higher labeling intensity than did anti-DP1/2. 

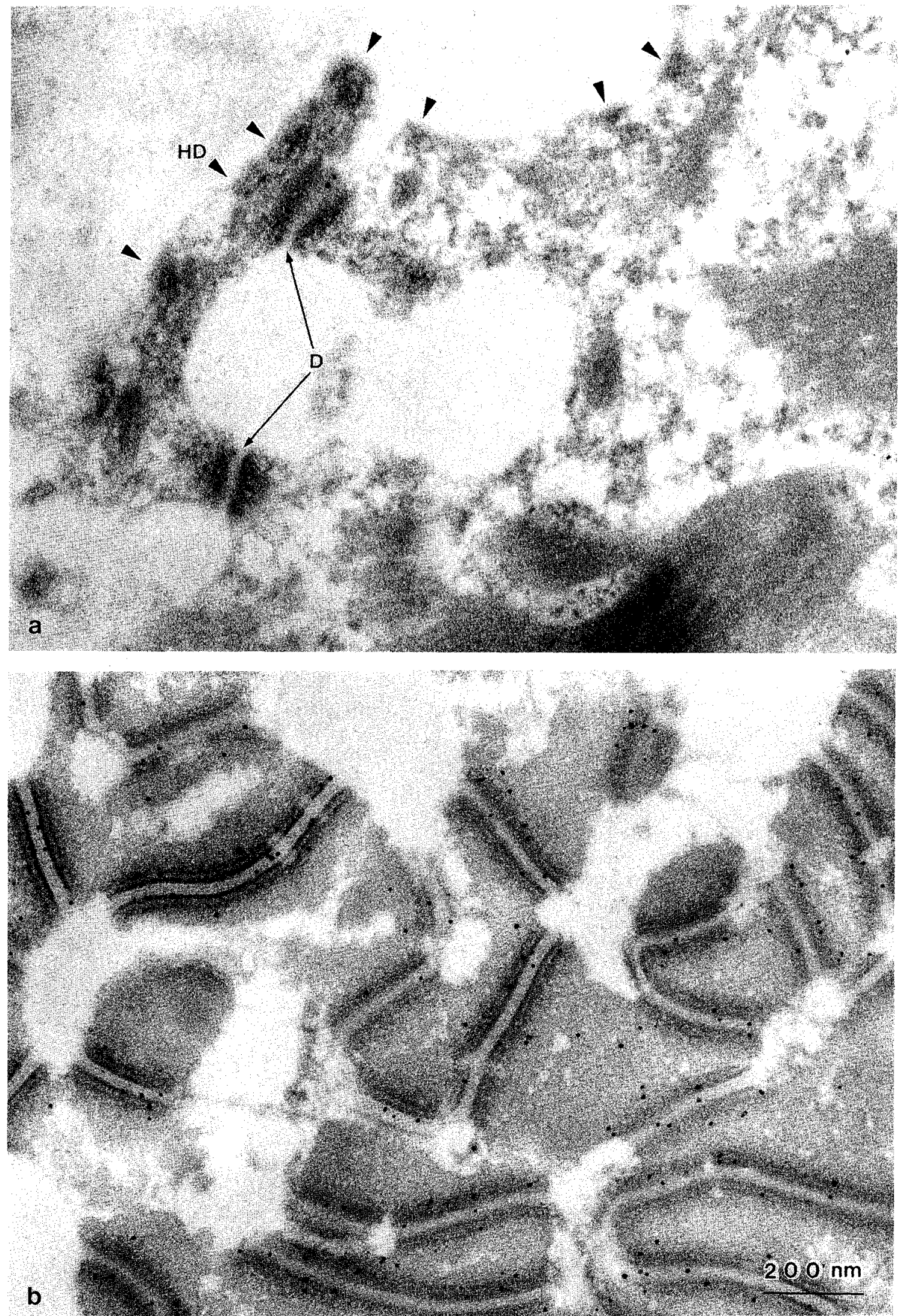

Fig. 2-a, b. 

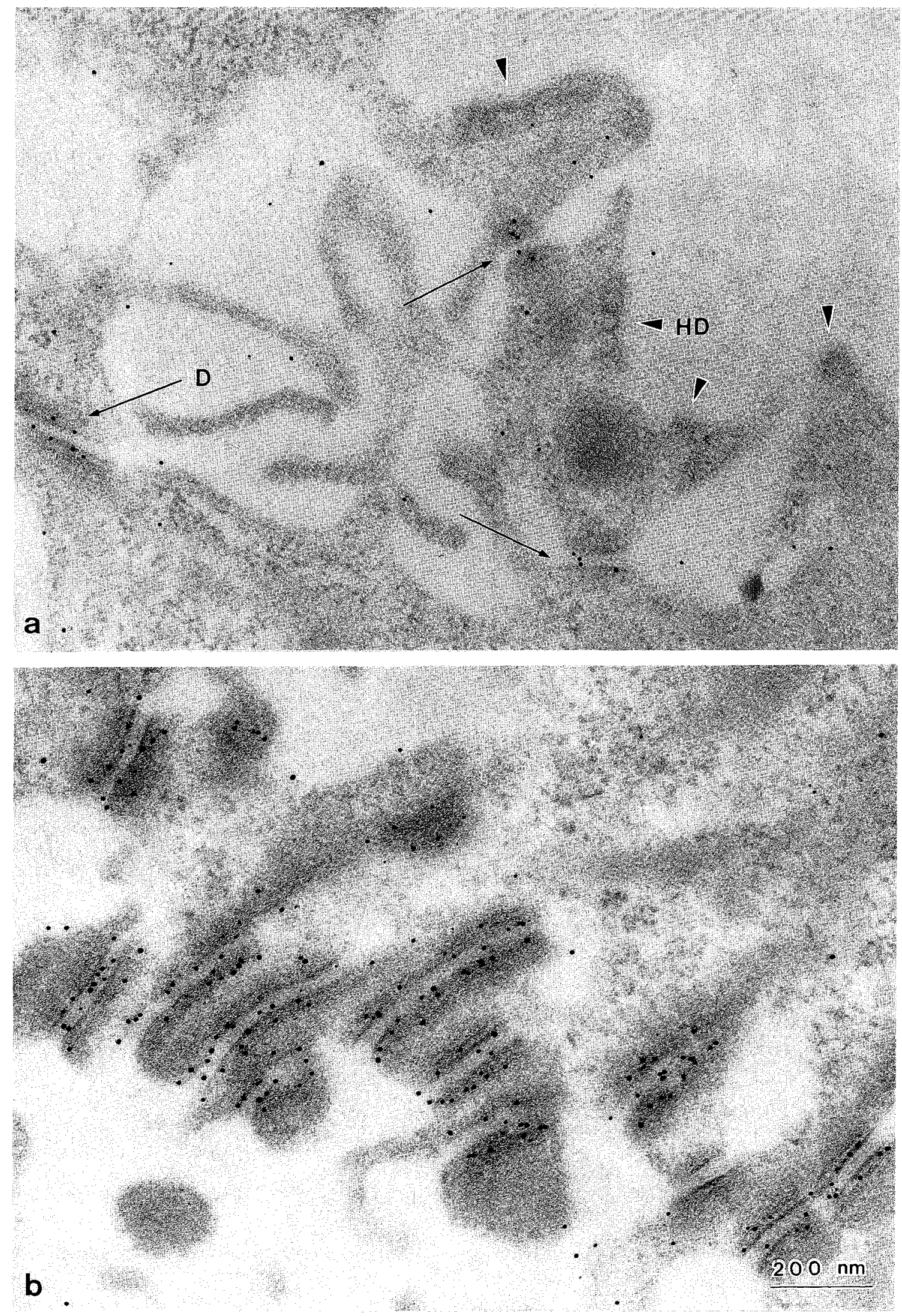

Fig. 3-a, b. 

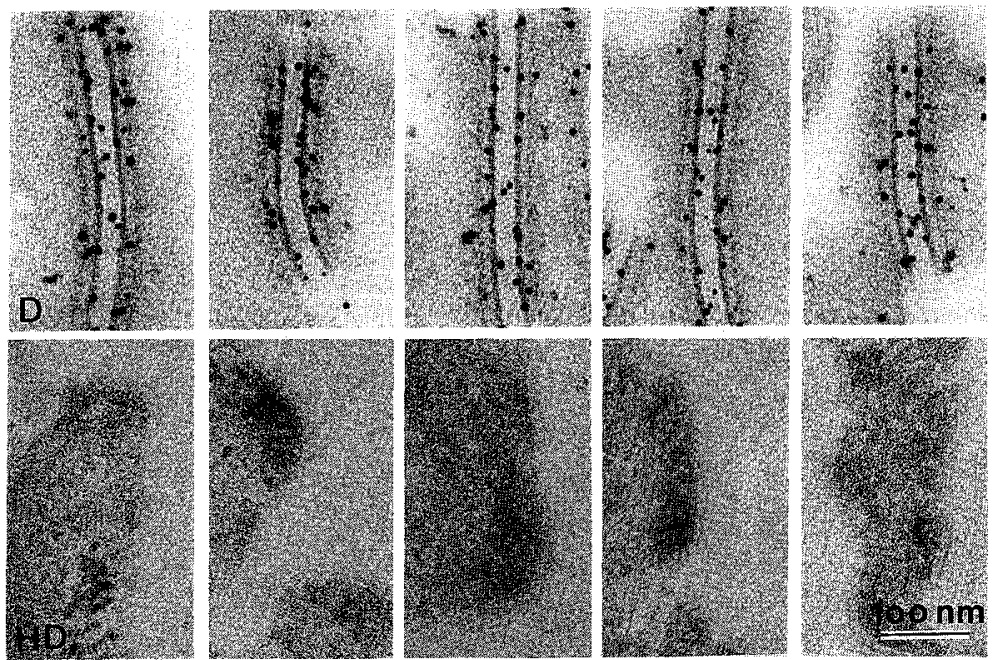

Fig. 4. Selected immunoelectron micrographs of desmosomes (D) and hemidesmosomes (HD). Note the same tendency as in Fig. 2.

The contrast between the negative reaction at the hemidesmosomes and the positive reaction at the desmosomes was clear and unmistakable, as shown in Fig. 4. The localization pattern of DG-type antigens, which are other major desmosomal proteins, was quite similar to that of DP-type antigens. Anti-DG1 (Fig. 5) and antiDG2/3 (Fig. 6) exhibited almost negative staining at hemidesmosome regions, while in the desmosomal regions, staining localized not only at the plaque, but also at the intercellular region.

Quantitative comparison. The above localization pattern was obtained by qualitative observation confirmed by quantitative comparison. As shown in Fig. 7, all of the specific reactions with four desmosomal antibodies showed distinct positive reactions at the desmosomes, and a contrasting negligible value at the hemidesmosomes.

\section{DISCUSSION}

In the experiment, we obtained a consistently negative reaction at the hemidesmosomes with four antibodies to bovine desmosomal antigens, including two DP types and two DG types. Although the results obtained with DP1/2 and with DP3 were basically similar to those of Jones et al. (6) and of Cowin et al. (3), respectively, we could not detect any positive staining of the hemidesmosomes with the antibody to DP1/2, as was reported in several previous papers $(10,11)$. According to Jones et al. (6), as well as to Yokoo et al. (24), the basement membrane zone where hemidesmosomes should be located was not stained by immunofluorescence with the antiserum to DP. On the contrary, Muller and Franke reported strong positive staining at the hemidesmosome plaque of multilayered epithelia or of the simple epithelia using the immunoperoxidase technique with anti-DP1/2 (11). Although the density of labeling was less than that seen at the desmosomes, relatively weak positive labeling at the hemidesmosomes was obtained by the immuno-gold method with anti-DP $1 / 2$ by other investigators as well (10).

To explain the above contradiction, the next two different types of preposition on the molecular organization of the hemidesmosome and the desmosome should be distinguished. One suggests a hypothetical state of molecular organization in which there are no molecular differences quantitatively or quantitatively between the hemidesmosome and the desmosome. The other offers a hypothetical state of the molecular organization as a heteroconstitutive state in which the number of molecules and/or primary structure of desmosomal antigens differ qualitatively or quantitatively in the hemidesmosome and the desmosome. The first hypothesis leads us to view the above contradiction as caused by a technical problem in detection, such as an immunochemical property of the antibody or "masking" of the desmosomal antigens. However, it is unthinkable that these different staining intensities result from immunocytochemical labeling with the same antibody for the same number and kind of molecules. In the same tissue of the

Fig. 2-a, b. Immunoelectron micrographs of bovine nasal epidermis labeled with anti-DP1/2 antibody followed by PAG. a: basal region; b: spinosum region. Gold particles can be observed only in the areas of the desmosomal plaques. Note no labeling of hemidesmosome (HD) in contrast to positive labeling of desmosomes (D) in the same microscopiec field (a).

Fig. 3-a, b. Immunoelectron micrographs of bovine nasal epidermis labeled with anti-DP3 antibody followed by PAG. a: basal region; b: spinosum region. 

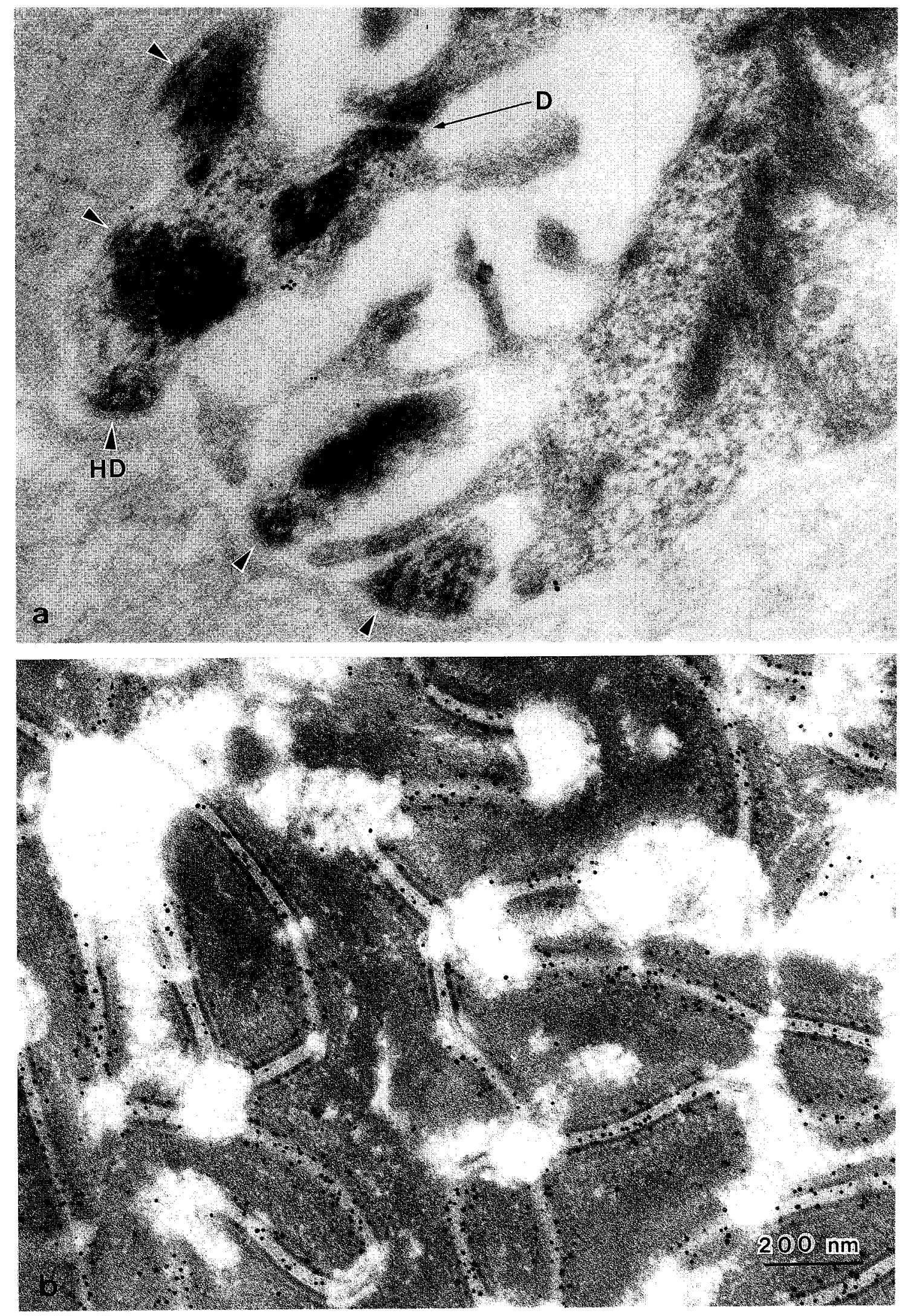

Fig. 5-a, b. 

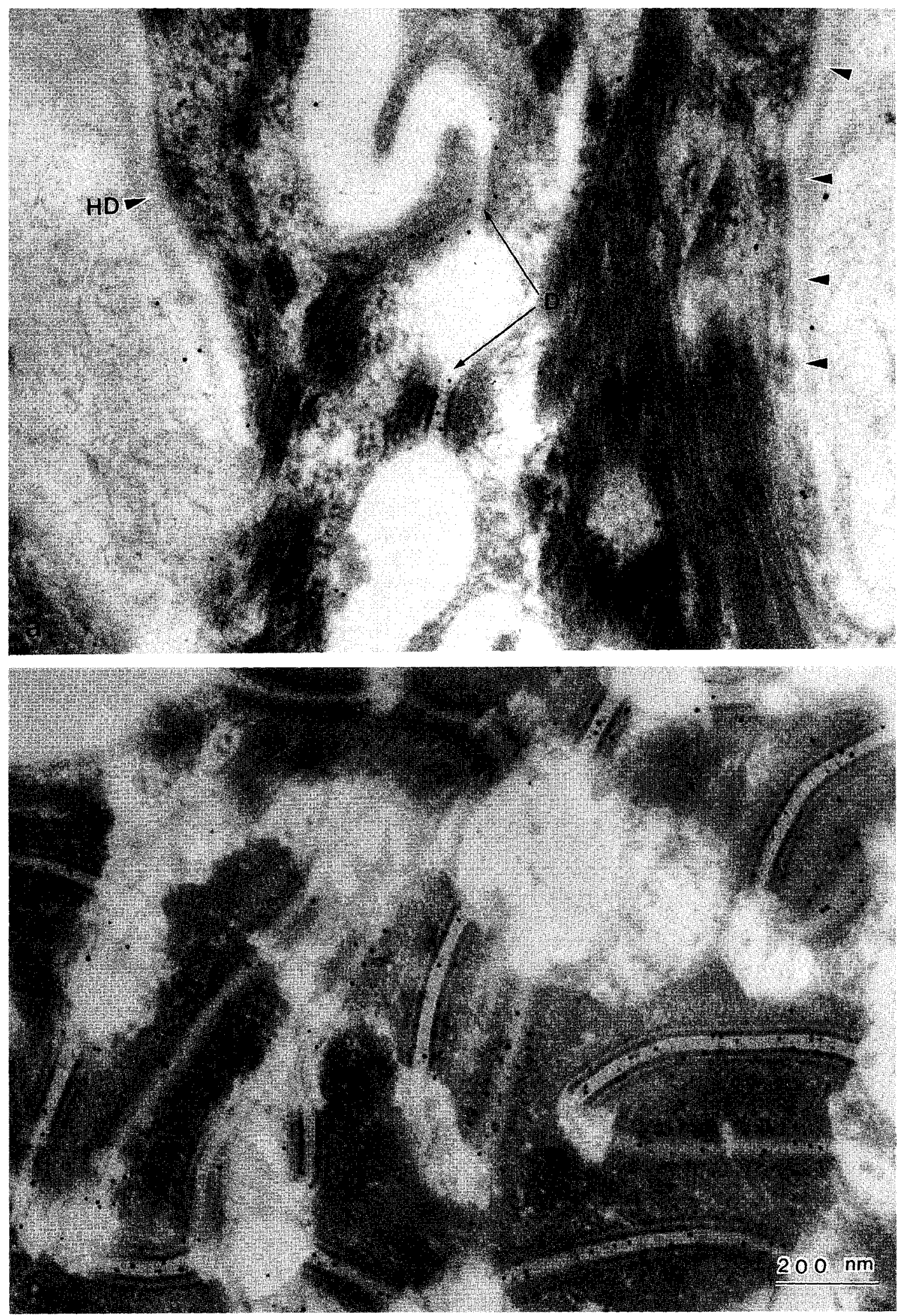

Fig. 6-a, b. 


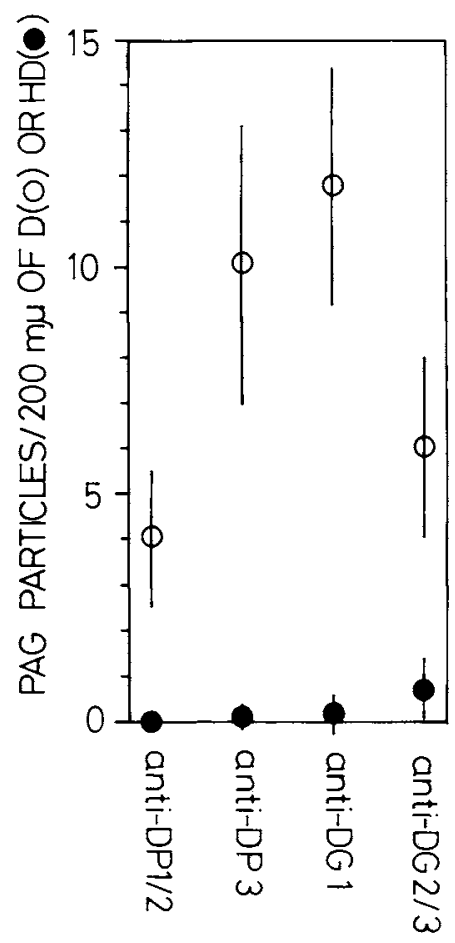

Fig. 7. Quantitative comparison of labeling intensity with four desmosomal antisera between desmosomes (open circles) and hemidesmosomes (solid circles).

same animal, another problem of masking is a very serious point in the fields of immunocytochemistry. The chemical meaning of masking includes the chemical condification of proteins by the plastic monomer or a steric hindrance of the epitopes in an intramolecular or intermolecular organization. The first reason given here cannot explain the contradiction because chemical modification is not restricted to the special proteins. Both the desmosomal antigens and the hemidesmosomal antigens can be randomly modified by plastic monomer. Steric hindrance is also unsatisfactory as an explanation, regardless of whether or not it were intramolecular or intermolecular. We used a postembedding labeling procedure. In this technique, specific labeling might be caused just on the surface of the ultrathin sections. At least within one type of protein organization, the intensity should be proportional to the number of epitopes per unit area, because even if "masking" did occur, the cutting procedure would act effectively to release the epitopes from that masking. The first hypothesis, then, can be disregarded because of its inconsistency with the experimental data.
If we assumed the second hypothesis of molecular organization, the major question about the labeling intensity of DP $1 / 2$ is assigned to the quality rather than quantity of the DP1/2 antigens, for the reasons stated below. DP1/2 are major proteins which constitute desmosomal plaques. If these plaque proteins are absent, electron-dense plaques can disappear, otherwise displacing other major desmosomal antigens. Hence, the relative amount of major plaque proteins will be roughly constant whether at desmosomes or at hemidesmosomes. The presence of the trace amount of DP $1 / 2$ at the plaque of hemidesmosomes might be disregarded.

For the above reasons, the qualitative difference of the antigenic properties between desmosomal and hemidesmosomal plaque proteins seems to be conceivable.

Recently, a cDNA sequence coding for a portion of the 230-KDa human BP antigen was reported (22), and comparison of the amino acid sequences with DP1/2 and $\mathrm{BP}$ antigen was carried out (5). According to the analysis, significant homology between a portion of the $\mathrm{BP}$ sequence and the three domains in the carboxy terminus of the DP $1 / 2$ was recognized. By virtue of this finding, it is possible to speculate that some of the polyclonal antibodies prepared against DP $1 / 2$ recognize the common domains between the DP1/2 and the BP antigens. However, for the reasons stated below, this similarity in the plaque proteins does not always support the traditional concept that the hemidesmosome is one half of the desmosome. The cell-to-substratum adhesion is not mediated by plaque proteins which have no transmembrane domains. Concerning the molecular organization of the desmosomal glycoproteins, the presence of transmembrane domains was suggested by a quantitative analysis of immunoelectron microscopy $(18,23)$, and by a sequential analysis of amino acids (9). To our great interest, the later data indicated a homology between DG1 and cadherin. Although no data have been reported on the dimeric homopolymer formation of cadherins, a homophilic interaction seems to be acceptable as a possible molecular mechanism of cell-tocell adhesion mainly because of the study on transfected $\mathrm{L}$ cells by exogenous cadherin cDNA $(12,15)$. Even if DG molecules were to localize in the hemidesmosomal region, it would still be impossible to make a homophilic binding of DG molecules at the basal region of the cells. Actually in the case of the two desmosomal glycoproteins (DG1, DG2/3), no distinct positive labeling at the hemidesmosomes was detected.

Recent studies on the immunocytochemical analysis of hemidesmosomes with monoclonal antibodies also

Fig. 5-a, b. Immunoelectron micrographs of bovine nasal epidermis labeled with anti-DG1 antibody followed by PAG. a: basal region; b: spinosum region.

Fig. 6-a, b. Immunoelectron micrographs of bovine nasal epidermis labeled with anti-DG2/3 antibody followed by PAG. a: basal region; b: spinosum region. 
support our results $(1,6,7,13,14)$. According to the serial experiments, none of the monoclonal antibodies which recognized hemidesmosomal antigens recognized the desmosomal constituents.

Our results, as well as previous results from studies using monoclonal antibodies directed against hemidesmosomes, as well as on the biochemical identification of hemidesmosomal constituents (17), suggest that the molecular constituents and functional meaning of hemidesmosomes are different from those of the desmosomes.

Acknowledgements. We would like to thank Dr. Sadaki Yokota (University of Yamanashi Medical School) for preparing the PAG complexes. We also thank Dr. Stephen M. Cohen for his assistance in preparing the antisera.

\section{REFERENCES}

1. Aiba, S., Masuko, T., Yagita, H., Hashimoto, Y., and TAGAMI, H. (1987). Monoclonal antibody AHS-7 defines a specific basement membrane antigen localized to the hemidesmosome zone. Acta Derm. Venereol. Stockn., 67: 399-405.

2. Cowin, P., Kappreli, H.-P., and Franke, W.W. (1985). The complement of desmosomal plaque proteins in different cell types. J. Cell Biol., 101: 1442-1454.

3. Cowin, P., Kapprell, H.-P., Franke, W.W., Tamkun, J., and HYNes, R.O. (1986). Plakoglobin: a protein common to different kinds of intercellular adhering junctions. Cell, 46: 1063-1073.

4. Giudice, G.J., Cohen, S.M., Patel, N.H., and Steinberg, M.S. (1984). Immunochemical comparison of desmosomal components from several bovine tissues. $J$. Cell. Biochem., 26: $35-45$.

5. Green, K.J., Parry, D.A.D., Stenert, P.M., Virata, M.L.A., Wagner, R.M., ANGS, B.D., and Nilles, L.A. (1990). Structure of the human desmoplakins. Implications for function in the desmosomal plaque. J. Biol. Chem., 265: 2603-2612.

6. Jones, J.C.R., Yokoo, K.M., and Goldman, R.D. (1986). Is the hemidesmosome a half desmosome? An immunological comparison of mannalian desmosomes and hemidesmosomes. Cell Motil. Cytoskel., 6: 560-569.

7. Jones, J.C.R. (1987). Characterization of components of the hemidesmosome (HD). J. Cell Biol., 105: 263a (abstract).

8. Kelly, D.E. (1966). Fine structure of desmosomes, hemidesmosomes, and an adepidermal globular layer in developing newt epidermis. $J$. Cell Biol., 28: 51-72.

9. Косh, P.J., Walsh, M.J., Schmerz, M., Goldschmidt, M.D., Zimbelmann, R., and Franke, W.W. (1990). Identification of desmoglein, a constitutive desmosomal glycoprotein, as a member of the cadherin family of cell adhesion molecules. Eur. $J$.
Cell Biol., 53: 1-12.

10. Mlller, K., Mattey, D., Measures, H., Hopkins, C., and GarroD, D. (1987). Localization of the protein and glycoprotein components of bovine nasal epithelial desmosomes by immunoelectron microscopy. EMBO J., 6: 885-889.

11. Mueller, H. and Franke, W.W. (1983). Biological and immunological characterization of desmoplakins I and II, the major polypeptides of the desmosomal plaque. J. Mol. Biol., 163: 647-671.

12. Nagafuchi, A., Shirayoshi, Y., OKazaki, K., and Takeichi, M. (1987). Transformation of cell adhesion properties by exogenously induced E-cadherin cDNA. Nature, 329: 341-405.

13. Negishi, A., Momose, F., Shioda, S., Takagi, T., Sasaki, S., and SEGAWA, A. (1989). A monoclonal antibody that recognizes hemidesmosomes but not desmosomes. Cell Struct. Funct., 14: 909 (abstract).

14. Nishizawa, Y. and Owaribe, K. (1989). Characterization of hemidesmosomal components by monoclonal antibodies. Cell Struct. Funct., 14: 909 (abstract).

15. NoSE, A., NAGAfUCHI, A., and TAKeIChI, M. (1988). Expressed recombinant cadherins mediate cell sorting in model systems. Cell, 54: 993-1001.

16. OHGA, R. and SHIDA, H. (1989). Comparative analysis on the molecular organization of desmosomal proteins in different tissues. Cell Struct. Funct., 14: 910 (abstract).

17. Owaribe, K., Nishizawa, Y., Stumpe, S., and Franke, W.W. (1989). Isolation of hemidesmosomes from bovine corneal epitherial cells. Cell Struct. Funct., 14: 908 (abstract).

18. Shida, H., Cohen, S.M., Givdice, G.J., and Steinberg, M.S. (1983). Quantitative electronmicroscopic immunocytochemistry of desmosomal antigens. J. Cell Biol., 97: 85a (abstract).

19. ShidA, H. and Steinberg, M.S. (1985). Comparison between the desmosome and hemidesmosome on the localization of desmosomal antigens. Cell Struct. Funct., 10: 545 (abstract).

20. Shida, H., Shida, M., and Ohga, R. (1987). A model experiment to develop a high resolving power analysis of immunoelectron microscopy. J. Electron Microsc., 36: 361-367.

21. SHIDA, H. and OHGA, R. (1990). Effect of resin use in the postembedding procedure on immunoelectron microscopy of membranous antigens, with special reference to sensitivity. $J$. Histochem. Cytochem., 38: 1687-1691.

22. Stanley, J.R., Tanaka, T., Mueller, S., Klaus-Kovtun, V., and RoOP, D. (1988). Isolation of complementary DNA for bullous pemphigoid antigen by use of patients' autoantibodies. J. Clin. Invest., 82: 1864-1870.

23. Steinberg, M.S., Shida, H., Giudice, G.J., Shida, M., Patel, N.H., and BLASCHUK, O.W. (1987). On the molecular organization, diversity and functions of desmosomal proteins. In Junctional complexes of epithelial cells (Ciba Foundation Symposium 125; G. Bock and S. Clark eds.). John Wiley \& Sons. U.K., pp 3-25.

24. Yokoo, K.M., Jones, J.C.R., and Goldman, R.D. (1985). Is the hemidesmosome a half-desmosome? $J$. Cell Biol., 101: 305a (abstract).

(Received for publication, January 31, 1991

and in revised form, March 4, 1991) 